\title{
Mutational screening of the USH2A gene in Spanish USH patients reveals 23 novel pathogenic mutations
}

Gema Garcia-Garcia', Maria J Aparisi ${ }^{1}$, Teresa Jaijo 1,2, Regina Rodrigo ${ }^{1}$, Ana M Leon², Almudena Avila-Fernandez ${ }^{2,3}$, Fiona Blanco-Kelly ${ }^{3}$, Sara Bernal ${ }^{2,4}$, Rafael Navarro ${ }^{5}$, Manuel Diaz-Llopis ${ }^{6}$, Montserrat Baiget ${ }^{2,4}$, Carmen Ayuso ${ }^{2,3}$, Jose M Millan ${ }^{1,2,7^{*}}$ and Elena Aller ${ }^{1,2}$

\begin{abstract}
Background: Usher Syndrome type II (USH2) is an autosomal recessive disorder, characterized by moderate to severe hearing impairment and retinitis pigmentosa (RP). Among the three genes implicated, mutations in the USH2A gene account for $74-90 \%$ of the USH2 cases.

Methods: To identify the genetic cause of the disease and determine the frequency of USH2A mutations in a cohort of 88 unrelated USH Spanish patients, we carried out a mutation screening of the 72 coding exons of this gene by direct sequencing. Moreover, we performed functional minigene studies for those changes that were predicted to affect splicing.

Results: As a result, a total of 144 DNA sequence variants were identified. Based upon previous studies, allele frequencies, segregation analysis, bioinformatics' predictions and in vitro experiments, 37 variants (23 of them novel) were classified as pathogenic mutations.

Conclusions: This report provide a wide spectrum of USH2A mutations and clinical features, including atypical Usher syndrome phenotypes resembling Usher syndrome type I. Considering only the patients clearly diagnosed with Usher syndrome type II, and results obtained in this and previous studies, we can state that mutations in USH2A are responsible for $76.1 \%$ of USH2 disease in patients of Spanish origin.
\end{abstract}

Keywords: Usher Syndrome, USH2A, Mutations, Sequence Variants

\section{Disease Name and Definition Usher syndrome}

Usher syndrome (USH) is an autosomal recessive disease characterized by hearing loss, retinitis pigmentosa $(\mathrm{RP})$, and, in some cases, vestibular dysfunction. It is clinically and genetically heterogeneous and is the most common cause underlying deafness and blindness of genetic origin. Clinically, USH is divided into three types. Usher type I (USH1) is the most severe form and is characterized by severe to profound congenital deafness, vestibular areflexia, and prepubertal onset of progressive RP. Type II (USH2) displays moderate to severe

\footnotetext{
* Correspondence: millan_jos@gva.es

'Grupo de Investigación en Enfermedades Neurosensoriales. Instituto de Investigación Sanitaria IIS-La Fe, Valencia, Spain

Full list of author information is available at the end of the article
}

hearing loss, absence of vestibular dysfunction, and later onset of retinal degeneration. Type III (USH3) shows progressive postlingual hearing loss, variable onset of $\mathrm{RP}$, and variable vestibular response. To date, five USH1 genes have been identified: MYO7A (USH1B), CDH23 (USH1D), PCDH15 (USH1F), USH1C(USH1C), and USH1G(USH1G). Three genes are involved in USH2, namely, USH2A (USH2A), GPR98 (USH2C), and DFNB31 (USH2D). USH3 is rare except in certain populations, and the gene responsible for this type is USH3A.

\section{Background}

Usher syndrome (USH) is an autosomal recessive disease characterized by the association of hearing loss and visual impairment due to retinitis pigmentosa (RP), with or without vestibular dysfunction. It is the most

\section{(Ciomed Central}


frequent cause of concurrent deafness and blindness of genetic origin and its general prevalence ranges from 3.3 to 6.4 per 100.000 live births [1]. In Spain, the estimation is 4.2/100.000 [2].

USH is clinically and genetically heterogeneous. Three clinical forms are distinguished: USH1, USH2 and USH3 and nine genes have been identified responsible so far. Five causative genes have been reported for USH1: MYO7A, USH1C, CDH23, PCDH15 and USH1G. Three genes for USH2: USH2A, GPR98 and DFNB31. Meanwhile only one gene has been described for USH3: USH3A $[3,4]$.

USH2 appears to be the most common clinical form of the disorder, accounting for more than $50 \%$ of all Usher cases [5,6]. Among the three genes described for USH2, USH $2 A$ is the most commonly mutated gene. It is responsible for approximately 74-90\% of USH2 cases $[2,7]$. Mutations in $U S H 2 A$, are also responsible for atypical Usher syndrome and recessive non-syndromic RP $[8,9]$. The $U S H 2 A$ gene, located on chromosome $1 \mathrm{q} 41$ [10], was initially described as comprising 21 exons, encoding a protein of 1546 amino acids [11,12]. However, in 2004, van Wijk et al. (2004) identified 51 additional exons at the 3' end of USH2A [13]. The longest transcript consists of 72 exons, encoding a protein of 5202 amino acids. In addition, Adato et al. (2005), identified an alternative spliced exon 71 in mouse transcripts, expressed in the inner ear and well conserved in vertebrates [14]. The long isoform $b$ is characterized by containing a transmembrane region, followed by an intracellular domain with a PDZ-binding motif, which interacts with the PDZ domain of harmonin and whirlin, integrating USH2A into the USH protein network $[14,15]$.

Initially, studies of the $U S H 2 A$ gene covered just exons $2-21[11,16-18,8,12,19,20,9,21,22]$, but only $45-63 \%$ of the expected $U S H 2 A$ mutations were identified. Nevertheless, since the discovery of the long isoform, several mutational analyses of all 72 exons have been carried out in diverse populations [23-30]. As a result, many novel pathogenic mutations have been identified, including splicing mutations at non-canonical positions of splice sites [31]. However, the majority of these changes only appear in a few cases, with the exception of the common ancestral p.Glu767fs mutation, located at exon 13 , which is the most prevalent $U S H 2 A$ mutation in several populations [16].

In the present study we have performed an exhaustive mutational screening of the long isoform $b$ of $U S H 2 A$ to identify new patients with mutations in this gene, and to detect the second mutation in patients with one previously detected $U S H 2 A$ mutation. Some cases had previously been studied for exon 13 or for the 21 first $U S H 2 A$ exons $[19,9]$, or analyzed using the genotyping microarray for Usher syndrome (Asper Biotech, Tartu, Estonia; [32]). Furthermore, we have used in silico and in vitro analysis to evaluate the functional consequences on gene expression and protein function of several nucleotide changes.

\section{Materials and methods \\ Subjects}

Eighty-eight (88) unrelated Spanish patients diagnosed of Usher syndrome were included in this study. They were recruited from the Federación de Afectados de Retinosis Pigmentaria de España (FARPE) and also from the Ophthalmology and ENT Services of several Spanish Hospitals as part of a large-scale study on the genetics of Usher syndrome in the Spanish population.

On the basis of their clinical history and ophthalmologic, audiological, neurophysiological and vestibular tests, 58 of these families were clinically classified as USH2 while 11 displayed atypical Usher syndrome. Detailed clinical data could not be obtained for 19 patients and these remained as non classified (USHNC).

Previously, 40 of these 88 patients were studied for exon 13, while 24 were analysed for the first 21 exons of $U S H 2 A$ and 42 were analyzed with the genotyping microarray for Usher syndrome (Asper Biotech, Tartu, Estonia). At that time, the version of the array detected 429 previously described mutations in eight of the nine genes reported for the disease. As a result of these previous analyses, eighteen of them were found to carry one mutated allele, but the second mutation could not be detected. These mutations have been included in the statistical summaries presented herein. These patients were subjected to mutation screening of the exons that had not been analyzed. In the remaining patients, we carried out the study of exons 2-72 (including the alternatively spliced exon 71).

When DNA samples from patients' relatives were available, we carried out a segregation analysis.

One hundred unrelated individuals of Spanish origin without hearing loss or RP family history were screened as controls to evaluate the frequency of the mutations found in the patient sample.

\section{Mutation analysis}

Genomic DNA from patients and controls was extracted from peripheral blood samples following standard protocols. The coding exons and flanking intronic sequences of USH $2 A$ were amplified by PCR using primers and conditions described by Aller et al. $(2004 ; 2006)[9,23]$. The amplified DNA fragments were analysed by direct sequencing using the Big Dye Terminator v.3.1 kit (Applied Byosistems, Carlsbad, CA), and purified sequencing reactions were analysed in an ABI PRISM 3730 DNA analyzer (Applied Byosistems, Carlsbad, CA). 
The obtained sequences were compared with the consensus sequence NM_206933.2. The +1 position corresponds to A in the ATG translation initiation codon.

\section{Predictions of the pathogenic effect of missense variations}

To predict whether a rare missense variant is deleterious, we used the combined results of three different computer algorithms:

-Sort Intolerant From Tolerant (SIFT) (available at http://sift.jcvi.org) uses sequence homology to predict whether a change is tolerated or deleterious.

-The polymorphism phenotyping program, PolyPhen (available at http://genetics.bwh.harvard.edu/pph/) uses sequence conservation, structure and SWISS-PROT annotation to characterize an amino acid substitution as benign, possibly deleterious or probably deleterious.

-Pmut (available at http://mmb2.pcb.ub.es:8080/PMut/ ) provides prediction by neural networks, which use internal databases, secondary structure prediction and sequence conservation. This program provides a binary prediction of "neutral" or "pathologic".

\section{Splice-site Prediction programs}

Intronic, isocoding and missense changes were analyzed using the programs NNSPLICE (http://fruitfly.org:9005/ seq_tools/splice.html), Human Splicing Finder (HSF) version 2.4 (http://www.umd.be/HSF/) and NetGene2 (http://www.cbs.dtu.dk/services/NetGene2/) in order to predict whether those changes could be affecting, creating or eliminating donor/acceptor splice sites.

\section{Minigene constructions and expression}

Minigene constructs were generated, using the exon trapping expression vector pSPL3. For each mutation, the exon and intronic flanking sequences were amplified from the patient's DNA, using the High Fidelity Phusion polymerase (Finnzymes, Espoo, Finland). Amplicons were inserted between the XhoI/NheI and XhoI/BamHI restriction sites for the variants p.E2496E and p.V382M, respectively, using T4 DNA ligase (Invitrogen Corporation, Carlsbad, CA). The p.V382M mutation was generated by site-directed mutagenesis. All vectors were confirmed by direct sequencing. The minigene constructs were transfected into COS-7 cells as described before [33]. RNA extraction and RT-PCR analysis was perfomed as previously described [34,31]. Missplicing percentages were measured using the Alpha Imager 2200 (version 3.1.2) software (AlphaInnotech Corporation, San Francisco, CA, USA).

\section{Results}

The molecular analysis of the $U S H 2 A$ gene in 88 unrelated USH Spanish patients revealed 37 different pathogenic mutations. Among these, a total of 23 mutations were novel (See Tables 1, 2 and 3). At least one pathogenic mutation was found in 43 out of 88 unrelated patients (48.9\%). Thirty-three patients were classified as USH2, five as USHA (atypical Usher syndrome) and five as USHNC (Usher syndrome non classified). In 25 out of these 43 cases the two causative mutations were detected $(58.1 \%)$, five patients were homozygous and 20 compound heterozygous. Detailed clinical manifestations of these 25 patients and 3 additional patients with one pathogenic and one probably pathogenic mutation (UV3; likely to be pathogenic but cannot formally be proven) are summarized in Table 4 .

In this study, a total of 144 variants were detected: 25 were truncating mutations and five were splice-site mutations (located at the conserved AG/GT dinucleotides of the splice site). The pathogenic effect of these variants is clear. But, in addition, 48 missense, 20 silent and 46 intronic variants were identified. According to previous studies, allele frequencies, segregation analysis, bioinformatics' predictions and in vitro experiments, the missense, silent and intronic changes were classified into 4 different categories: pathogenic, possibly pathogenic (UV3), possibly non-pathogenic (possibly neutral, UV2) and non-pathogenic (neutral). (See Tables 1, 2,3 and 5).

\section{Missense variants}

Fourty-eight missense variants were identified (See Table 2). Twenty-nine were considered as non-pathogenic because all of them were already described as non-pathogenic in other studies [https://grenada.lumc. nl/LOVD2/Usher_montpellier, [35]].

Nine nucleotide changes were classified as possibly non-pathologic (UV2). p.G713R, p.S841Y, p.S2196T, p. S2639P, p.G4692R and p.K5026E have already been reported in other works and categorized as possibly non-deleterious or of unknown pathogenecity [https:// grenada.lumc.nl/LOVD2/Usher_montpellier, [35]]. Meanwhile, p.N2377S, p.N2394K and p.E4921K have not been described previously, so they were analyzed with the three sequence analysis programs (SIFT, Polyphen and PMUT). None of those changes was predicted to be clearly deleterious (See Table 6).

Four missense variants were classified as possiblypathogenic (UV3). The variants p.R303H and p.Y1992C were described previously [https://grenada.lumc.nl/ LOVD2/Usher_montpellier, [35]]. The novel change p. N3894D was not found in 200 control alleles and the segregation analysis proved that it co-segregates with the disease. However, only one program considered it as clearly pathogenic (See Table 6). The new p.V382M change, which affects the first base of exon 7 , was not found in control samples and it was predicted to slightly affect splicing. The minigene assays only revealed a mild 
Table 1 USH2A truncating and splice-site mutations

\begin{tabular}{|c|c|c|c|c|c|}
\hline Nucleotide change & Exon & Predicted effect & Predicted pathology & No. of alleles & References \\
\hline \multicolumn{6}{|l|}{ Nonsense mutations } \\
\hline$c .820 \mathrm{C}>\mathrm{T}$ & 5 & p.R274X & + & 2 & Present study \\
\hline c. $1518 \mathrm{~T}>\mathrm{A}$ & 8 & p.Y506X & + & 1 & Bernal et al., 2005 \\
\hline c.3883C > T & 18 & p.R1295X & + & 1 & Dreyer et al., 2000 \\
\hline c. $4474 \mathrm{G}>\mathrm{T}$ & 21 & p.E1492X & + & 2 & Bernal et al., 2005 \\
\hline c. $4645 \mathrm{C}>\mathrm{T}$ & 22 & p.R1549X & + & 1 & Baux et al., 2007 \\
\hline c.7854G > C & 41 & p.W2618X & + & 1 & Present study \\
\hline c. $9753 \mathrm{~T}>\mathrm{A}$ & 50 & p.C3251X & + & 2 & Present study \\
\hline c. $10102 C>T$ & 51 & p.Q3368X $x^{a}$ & + & 1 & Present study \\
\hline c. $10759 C>T$ & 55 & p.Q3587X & + & 2 & Baux et al., 2007 \\
\hline c. $11146 C>T$ & 57 & p.Q3716X & + & 1 & Present study \\
\hline c. $14175 G>A$ & 65 & P.W4725X & + & 1 & Present study \\
\hline \multicolumn{6}{|l|}{ Deletions and insertions } \\
\hline c.918_919insGCTG & 6 & p.S307AfsX17 & + & 1 & Present study \\
\hline c.1214delA & 7 & p.N405lfsX3 & + & 5 & Bernal et al., 2005 \\
\hline c.1629_1645del & 10 & p.F543LfsX2 & + & 1 & Present study \\
\hline c.2299delG & 13 & p.E767Sfs X21 & + & 8 & Eudy et al., 1998 \\
\hline c.5278delG & 26 & p.D1760MfsX10 & + & 1 & Present study \\
\hline c.5540_5541dup & 27 & p.N1848X & + & 1 & Present study \\
\hline c.6319_6324delinsTAAA & 32 & p.V2107X & + & 1 & Present study \\
\hline c.8890dupT & 45 & p.W2964LfsX89 & + & 1 & Present study \\
\hline c.8954delG & 45 & p.G2985AfsX3 & + & 1 & Present study \\
\hline c.9261delT & 47 & p.E3088KfsX9 & + & 1 & Present study \\
\hline c.10272_10273dupTT & 52 & p.C3425FfsX4 & + & 1 & Aller et al., 2006 \\
\hline c.11566delA & 60 & p.S3856VfsX28 & + & 1 & Present study \\
\hline c.12093delC & 62 & p.Y4031X & + & 1 & Present study \\
\hline c.13140delA & 63 & p.V4381CfsX10 & + & 1 & Present study \\
\hline \multicolumn{6}{|l|}{ Splice-site mutations } \\
\hline$c .1328+1 G>T$ & IVS7 & Ex7 splice defect & + & 1 & Present study \\
\hline c. $1841-2 A>G$ & IVS10 & Ex11 splice defect & + & 2 & Bernal et al., 2003 \\
\hline c. $11548+2 \mathrm{~T}>\mathrm{G}$ & IVS59 & Ex59 splice defect & + & 1 & Present study \\
\hline c. $12067-2 A>G$ & IVS61 & Ex62 splice defect & + & 4 & Auslender et al., 2008 \\
\hline c. $15053-1 \mathrm{G}>\mathrm{A}$ & IVS69 & Ex70 splice defect & + & 1 & Present study \\
\hline
\end{tabular}

+: Denotes pathogenic mutations.

No. of alleles: Number of alleles identified in patients.

${ }^{a}$ These two mutations are allelic.

Novel pathogenic mutations described in this study are in bold.

increase of the transcript excluding exon 7 (Figure 1, band d) when the variant was present, in comparison to the wild-type sequence.

Finally, six missense mutations were considered as pathogenic. p.C759F and p.C3267R were already described by others authors as damaging [https://grenada.lumc.nl/ LOVD2/Usher_montpellier, [35]]. p.C3358Y and p.P4818L were classified by McGee et al. (2010) [30] and the LOVDUSH Database as likely-pathogenic (UV3). However, in the present study, p.C3358Y was detected in a patient together with another nucleotide change (p.C3267R) and the segregation analysis confirmed that the mutations were not in the same allele. p.P4818L was detected in a patient together with two other mutations that directly or indirectly cause a truncated protein (p.Q3368X and c.5278delG). The segregation analysis confirmed that the deletion and the nonsense mutation were in cis and the missense variant was in the other allele and cosegregated with the disease. The segregation analyses support the damaging effect of p.C3358Y and p.P4818L, so we have considered them as pathogenic. p. 
Table 2 Missense changes in USH2A

\begin{tabular}{|c|c|c|c|c|c|}
\hline Nucleotide change & Exon & Amino acid change & Predicted pathology & No. of alleles & Ref. \\
\hline c. $373 G>A$ & 2 & p.A125T & - & 81 & Dreyer et al., 2000 \\
\hline c.130G > A & 2 & p.G44R & + & 1 & Present Study \\
\hline c. $688 \mathrm{G}>\mathrm{A}$ & 4 & p.V230M & - & 3 & Dreyer et al., 2000 \\
\hline$c .908 \mathrm{G}>\mathrm{A}$ & 6 & p.R303 $\mathrm{H}^{\mathrm{e}}$ & UV3 & 1 & Yan et al., 2009 \\
\hline c.1144G > A & 7 & p.V382M ${ }^{b}$ & UV3 & 1 & Present Study \\
\hline c. $1434 G>C$ & 8 & p.E478D & - & 4 & Seyedahmadi et al., 2004 \\
\hline c. $1663 C>G$ & 10 & p.L555V & - & 2 & Bernal et al., 2003 \\
\hline C.1931A > T & 11 & p.D644V & - & 6 & Weston et al., 2000 \\
\hline$c .2137 G>C$ & 12 & P.G713R & UV2 & 2 & Dreyer et al., 2000 \\
\hline c. $2276 \mathrm{G}>\mathrm{T}$ & 13 & p.C759F & + & 3 & Dreyer et al., 2000 \\
\hline c. $2522 C>A$ & 13 & p.S841Y & UV2 & 1 & Jaijo et al., 2009 \\
\hline c. $4457 \mathrm{G}>\mathrm{A}$ & 21 & p.R1486K & - & 75 & Dreyer et al., 2000 \\
\hline C. $4714 C>T$ & 22 & p.L1572F & - & 7 & Dreyer et al., 2008 \\
\hline c.4994T > C & 25 & p.11665T & - & 31 & Kaiserman et al., 2007 \\
\hline C.5975A > G & 30 & p.Y1992C & UV3 & 1 & McGee et al., 2010 \\
\hline C.6317T > C & 32 & p.12106T & - & 117 & Aller et al., 2006 \\
\hline c.6506T > C & 34 & p.12169T & - & 85 & Aller et al., 2006 \\
\hline c. $6587 G>C$ & 34 & p.S2196T & UV2 & 2 & Jaijo et al., 2009 \\
\hline c. $6713 \mathrm{~A}>\mathrm{C}$ & 35 & p.E2238A & - & 1 & Aller et al., 2006 \\
\hline c. $6875 \mathrm{G}>\mathrm{A}$ & 36 & p.R2292H & - & 2 & Dreyer et al., 2008 \\
\hline c. $7130 \mathrm{~A}>\mathrm{G}$ & 38 & p.N2377S & UV2 & 1 & Present Study \\
\hline C. $7182 C>A$ & 38 & p.N2394K & UV2 & 1 & Present Study \\
\hline c.7506G > A & 40 & p.P2502P & - & 11 & Baux et al., 2008 \\
\hline c.7685T > C & 41 & p.V2562A & - & 1 & Dreyer et al., 2008 \\
\hline C.7915T > C & 41 & p.S2639P & UV2 & 3 & McGee et al., 2010 \\
\hline c. $8624 G>A$ & 43 & p.R2875Q & - & 9 & Aller et al., 2006 \\
\hline c. $8656 C>T$ & 43 & p.L2886F & - & 9 & Aller et al., 2006 \\
\hline$c .9262 G>A$ & 47 & p.E3088K & - & 1 & Dreyer et al., 2008 \\
\hline c. $.9296 \mathrm{~A}>\mathrm{G}$ & 47 & p.N3099S & - & 12 & Aller et al., 2006 \\
\hline c. $9343 \mathrm{~A}>\mathrm{G}$ & 47 & p.T3115A & - & 9 & Dreyer et al., 2008 \\
\hline c. $9430 \mathrm{G}>\mathrm{A}$ & 48 & p.D3144N & - & 8 & Aller et al., 2006 \\
\hline c.9595A > G & 49 & p.N3199D & - & 9 & Baux et al., 2007 \\
\hline C.9799T > C & 50 & p.C3267R & + & 5 & Aller et al., 2006 \\
\hline c. $10073 \mathrm{G}>\mathrm{A}$ & 51 & p.C3358Y & + & 1 & McGee et al., 2010 \\
\hline C. $10232 A>C$ & 52 & p.E3411A & - & 94 & Aller et al., 2006 \\
\hline c.10636G > A & 54 & p.G3546R & + & 4 & Present Study \\
\hline C. $11504 C>T$ & 59 & p.T3835I & - & 30 & Present Study \\
\hline c. $11602 A>G$ & 60 & p.M3868V & - & 34 & Aller et al., 2006 \\
\hline C. $11677 C>A$ & 60 & p.P3893T & - & 2 & Dreyer et al., 2008 \\
\hline c. $11680 \mathrm{~A}>\mathrm{G}$ & 60 & p.N3894D & UV3 & 1 & Present Study \\
\hline c. $12343 \mathrm{C}>\mathrm{T}$ & 63 & p.R4115C & - & 2 & van Wijk et al., 2004 \\
\hline c. $14074 \mathrm{G}>\mathrm{A}$ & 64 & p.G4692R & UV2 & 1 & McGee et al., 2010 \\
\hline c. $14453 \mathrm{C}>\mathrm{T}$ & 66 & p.P4818L & + & 1 & Aller et al., 2006 \\
\hline c. $14513 G>A$ & 66 & p.G4838E & - & 1 & McGee et al., 2010 \\
\hline c. $14543 G>A$ & 66 & p.R4848Q & - & 1 & McGee et al., 2010 \\
\hline c. $14761 G>A$ & 67 & p.E4921K & UV2 & 1 & Present Study \\
\hline c. $15076 \mathrm{~A}>\mathrm{G}$ & 70 & p.K5026E & UV2 & 1 & McGee et al., 2010 \\
\hline
\end{tabular}


Table 2 Missense changes in USH2A (Continued)

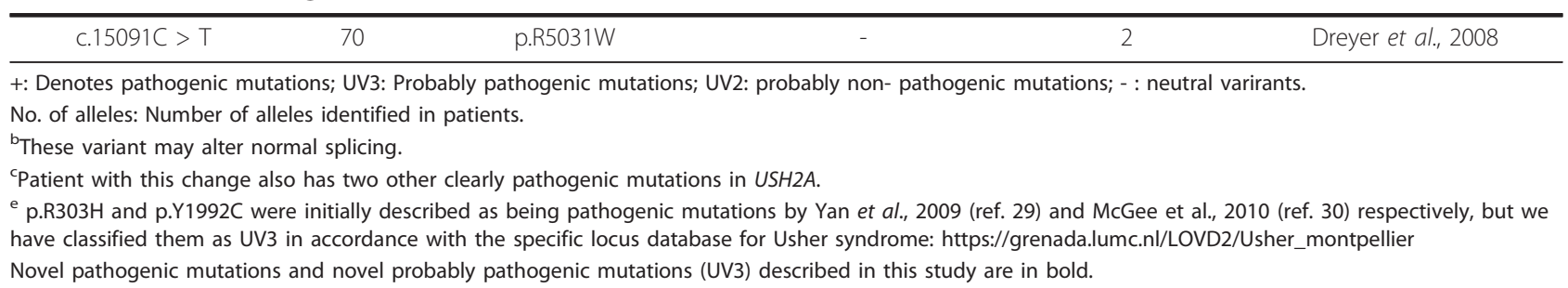

G44R and p.G3546R were novel; none of them was detected in 200 control alleles. The variant p.G44R was detected in a single patient and p.G3546R in three cases (one homozygous and two compound heterozygous cases); the results of the three computational analyses classified them as pathogenic (See Table 6).

\section{Silent variants}

We also identified 20 silent variants, 17 were previously described as neutral [[36], https://grenada.lumc.nl/ LOVD2/Usher_montpellier, [35]] and three were novel (See Table 3). Only the variant p.E2496E was categorized as pathogenic. It was not found in 200 control alleles and the segregation analysis confirmed that this mutation co-segregates with the disease. We detected this variant in trans in a patient who also had a premature stop codon. According to in silico analyses, it was predicted to create a de novo donor splice site (data not shown). The splicing alteration was confirmed using hybrid minigenes. The mutant construct generated a transcript that lacked the last 106 nucleotides of exon 40 (Figure 1, band b). This loss of nucleotides creates a new open reading frame, leading to a premature stop codon five amino acids downstream.

\section{Intronic variants}

Fourty-six intronic variants located at non-canonical positions of splice sites, of which 20 are novel, were detected

Table 3 Silent variants in USH2A

\begin{tabular}{|c|c|c|c|c|c|}
\hline Nucleotide change & Exon & Amino acid position & Predicted pathology & No. of alleles & Ref. \\
\hline c. $504 \mathrm{~A}>\mathrm{G}$ & 3 & p.T168T & - & 75 & Baux et al., 2008 \\
\hline c. $1179 A>G$ & 7 & p.Q393Q & - & 1 & Aller, 2008 \\
\hline c. 1419 C $>$ T & 8 & p.T473T & - & 36 & Dreyer et al., 2000 \\
\hline c.2109T > C & 12 & p.D703D & - & 7 & Weston et al., 2000 \\
\hline c.2256T > C & 13 & p.H752H & - & 3 & Dreyer et al., 2008 \\
\hline C.3945T > C & 18 & p.N1315N & UV2 & 3 & Present Study \\
\hline c.4371G > A & 20 & p.S1457S & - & 1 & Dreyer et al., 2000 \\
\hline c. $5031 \mathrm{C}>\mathrm{A}$ & 25 & p.G1671G & - & 22 & Aller et al., 2006 \\
\hline C. $5751 C>T$ & 28 & p.Y1917Y & UV2 & 1 & McGee et al., 2010 \\
\hline c. $7488 \mathrm{~A}>\mathrm{G}$ & 40 & p.E2496E ${ }^{b}$ & + & 1 & Present Study \\
\hline c.7506G > A & 40 & p.P2502P & - & 11 & McGee et al., 2010 \\
\hline c. $11736 G>A$ & 61 & p.E3912E & - & 2 & Dreyer et al., 2008 \\
\hline C.11907A > T & 61 & p.P3969P & - & 2 & Dreyer et al., 2008 \\
\hline C.11946A > T & 61 & p.L3982L & - & 29 & Dreyer et al., 2008 \\
\hline c. 12093 C > T & 62 & p.Y4031Y & - & 1 & Dreyer et al., 2008 \\
\hline c. $12612 A>G$ & 63 & p.T4204T & - & 129 & Dreyer et al., 2008 \\
\hline c. $12666 \mathrm{~A}>\mathrm{G}$ & 63 & p.T4222T & - & 58 & Dreyer et al., 2008 \\
\hline c.13191G > A & 63 & p.E4397E & - & 23 & Dreyer et al., 2008 \\
\hline c. $14481 C>T$ & 66 & p.A4827A & - & 1 & McGee et al., 2010 \\
\hline c. $14664 G>A$ & 67 & p.T4888T & UV2 & 1 & Present Study \\
\hline
\end{tabular}

+: Denotes pathogenic mutations; UV2: probably non- pathogenic mutations; - : neutral variants.

No. of alleles: Number of alleles identified in patients.

${ }^{\mathrm{b}}$ These variant may alter normal splicing.

'Patient with this change also has two other clearly pathogenic mutations in USH2A.

Novel pathogenic mutations described in this study are in bold. 
Table 4 Genotype-phenotype correlations of USH patients with both mutations found in this study

\begin{tabular}{|c|c|c|c|c|c|c|c|c|c|c|c|c|c|}
\hline Patient & Mutations & $\begin{array}{l}\text { Year } \\
\text { of } \\
\text { Birth }\end{array}$ & Diagnosis & $\begin{array}{c}\text { Age of } \\
\text { diagnosis }\end{array}$ & $\begin{array}{l}\text { Sensorineural } \\
\text { Hearing Loss }\end{array}$ & $\begin{array}{l}\text { Vestibular } \\
\text { Function }\end{array}$ & $\begin{array}{c}\text { Onset of } \\
\text { Night } \\
\text { Blindness }\end{array}$ & $\begin{array}{l}\text { Onset of } \\
\text { Visual Field } \\
\text { Loss }\end{array}$ & Visual Field & $\begin{array}{l}\text { Visual } \\
\text { Acuity }\end{array}$ & $\begin{array}{l}\text { Eye } \\
\text { Fundus }\end{array}$ & ERG & Cataracts \\
\hline RP1310* & $\begin{array}{l}\text { c. } 12067-2 A>G / \\
\text { c. } 12067-2 A>G\end{array}$ & & USHNC & & & & & & & & & & \\
\hline RP1274* & p.E1492X/p.E1492X & 1969 & USH2 & & $\begin{array}{c}\text { Moderate-severe } \\
\text { and stable }\end{array}$ & Normal $^{A}$ & & & & & 1 & & \\
\hline RP1633 & p.E1492X/p.E1492X & 1962 & USH2 & 25 & Since infancy & Normal $^{A}$ & 25 & 25 & Concentric loss & $0,2 / 0,4$ & 1 & $\begin{array}{l}\text { No } \\
\text { response }\end{array}$ & $\mathrm{BE}$ \\
\hline RP1607 & p.G3546R/p.G3546R & 1925 & USH2 & 33 & Mild-moderate & Normal $^{A}$ & 16 & 25 & $\begin{array}{c}\text { Marked } \\
\text { concentric loss }\end{array}$ & $0,6 / 0,6$ & 1 & $\begin{array}{l}\text { Moderate } \\
\text { alteration }\end{array}$ & $\mathrm{BE}$ \\
\hline RP1599* & c.1214delA/c.1214delA & 1980 & USH2 & & $\begin{array}{l}\text { Moderate, since } \\
\text { infancy }\end{array}$ & Normal $^{A}$ & 20 & 30 & $\begin{array}{c}\text { Moderate } \\
\text { concentric loss }\end{array}$ & $0,6 / 0,7$ & 1 & $\begin{array}{l}\text { Moderate } \\
\text { alteration }\end{array}$ & BE \\
\hline RP259 & c.1214delA/p.C3267R & 1974 & USHA & 19 & $\begin{array}{c}\text { Profound since } 6 \\
\text { years }\end{array}$ & $\begin{array}{c}\text { Vestibular } \\
\text { Dysfunction }^{A}\end{array}$ & 6 & & $\begin{array}{c}\text { Concentric } \\
\text { loss ( } 19 \text { years) }\end{array}$ & $\begin{array}{c}0,35 / \\
0,35(19 \\
\text { years) }\end{array}$ & 1 & $\begin{array}{l}\text { No } \\
\text { response }\end{array}$ & $\begin{array}{l}\text { No }(19 \\
\text { years })\end{array}$ \\
\hline RP1349 & c.2299delG/c.1214delA & 1954 & USH2 & & Moderate-severe & Normal $^{B}$ & 15 & 20 & $\begin{array}{c}\text { Marked } \\
\text { concentric loss }\end{array}$ & $\begin{array}{c}0,4 / 0,3 \\
\text { (30 years) }\end{array}$ & 2 & & \\
\hline RP1493 & c.2299delG/c.8890dupT & 1973 & USH2 & 30 & $\begin{array}{l}\text { Congenital, } \\
\text { moderate and } \\
\text { stable }\end{array}$ & Normal $^{B}$ & 29 & 29 & $\begin{array}{l}\text { Concentric loss } \\
\text { (at } 31 \text { years) }\end{array}$ & $\begin{array}{l}\text { Normal } \\
\text { (31 years) }\end{array}$ & 1 & $\begin{array}{c}\text { No } \\
\text { response } \\
\text { (31 years) } \\
\end{array}$ & $\begin{array}{l}\text { No }(31 \\
\text { years) }\end{array}$ \\
\hline RP1632 & c.2299delG/c.8954delG & 1961 & USH2 & & Since infancy & Normal $^{B}$ & 23 & 25 & & $<0,1 \mathrm{BE}$ & 2 & $\begin{array}{l}\text { No } \\
\text { response }\end{array}$ & BE \\
\hline RP1715* & $\begin{array}{c}\text { c.2299delG/ } \\
\text { c.1629_1645del }\end{array}$ & 1986 & USH2 & 22 & $\begin{array}{c}\text { Moderate and } \\
\text { stable since } 6 \text { years }\end{array}$ & Normal $^{B}$ & 16 & 20 & $\begin{array}{l}\text { Slight } \\
\text { concentric loss } \\
\text { (at } 25 \text { years) }\end{array}$ & $\begin{array}{l}\text { Normal } \\
\text { (25 years) }\end{array}$ & 1 & $\begin{array}{l}\text { No } \\
\text { response }\end{array}$ & No \\
\hline RP1775 & c.2299delG/p.R303H & 1961 & USHA & 30 & $\begin{array}{c}\text { Moderate since } 7 \\
\text { years and } \\
\text { progressive }\end{array}$ & & 23 & 22 & & & & & \\
\hline RP1618 & $\begin{array}{c}\text { p.C3267R/ } \\
\text { c.6319_6324delinsTAAA }\end{array}$ & 1964 & USH2 & & Severe-profound & Normal $^{A}$ & 30 & 30 & $\begin{array}{c}\text { Marked } \\
\text { concentric loss }\end{array}$ & $0,1 / 0,1$ & 2 & $\begin{array}{l}\text { No } \\
\text { response }\end{array}$ & $\mathrm{BE}$ \\
\hline RP1442* & p.C3267R/c.12093delC & 1962 & USH2 & 20 & $\begin{array}{l}\text { Congenital, } \\
\text { moderate and } \\
\text { stable }\end{array}$ & Normal $^{B}$ & 15 & 20 & $\begin{array}{l}\text { Concentric loss, } \\
5^{\circ} \text { (at } 43 \text { years) }\end{array}$ & $\begin{array}{c}0,5 / 0,2 \\
\text { (43 years) }\end{array}$ & 2 & & $\begin{array}{c}\text { LE (37 } \\
\text { years) RE } \\
\text { (43 years) } \\
\end{array}$ \\
\hline RP1703* & p.C3267R/p.C3358Y & 1936 & USHA & 50 & Since 64 years & & 50 & 55 & $\begin{array}{l}\text { Reduced (67 } \\
\text { years) }\end{array}$ & & 1 & & Yes \\
\hline RP1759 & p.C3267R/p.Y1992C\# & 1945 & USH2 & 62 & $\begin{array}{l}\text { Congenital, } \\
\text { moderate and } \\
\text { stable }\end{array}$ & Normal $^{B}$ & & 62 & & & & $\begin{array}{l}\text { Abnormal } \\
\text { response }\end{array}$ & $\begin{array}{l}\text { Yes }(62 \\
\text { years) }\end{array}$ \\
\hline RP1625* & $\begin{array}{c}\text { c.12067-2A > G/p. } \\
\text { R274X }\end{array}$ & 1976 & USH2 & 25 & Since infancy & Normal $^{A}$ & 19 & 24 & Concentric loss & $0,1 / 0,1$ & 2 & $\begin{array}{l}\text { No } \\
\text { response }\end{array}$ & $\mathrm{BE}$ \\
\hline RP1631 & c.1841-2A > G/p.R274X & 1976 & USH2 & & Since infancy & Normal $^{\mathrm{A}}$ & 28 & 28 & Concentric loss & $0,7 / 0,6$ & 1 & & No \\
\hline
\end{tabular}


Table 4 Genotype-phenotype correlations of USH patients with both mutations found in this study (Continued)

\begin{tabular}{|c|c|c|c|c|c|c|c|c|c|c|c|c|c|}
\hline RP951 & c.1214delA/p.C3251X & 1969 & USH2 & 25 & $\begin{array}{l}\text { Congenital, severe } \\
\text { and stable }\end{array}$ & Normal $^{B}$ & 25 & 18 & $\begin{array}{l}\text { Reduced }(23 \\
\text { years) }\end{array}$ & & 2 & $\begin{array}{c}\text { No } \\
\text { response } \\
\text { (30 years) }\end{array}$ & $\begin{array}{l}\text { No }(30 \\
\text { years) }\end{array}$ \\
\hline RP1558 & $\begin{array}{c}\text { p.R1549X/c.1328 + 1G } \\
>\mathrm{T}\end{array}$ & 1933 & USH2 & 43 & $\begin{array}{c}\text { Severe and } \\
\text { progressive since } \\
20 \text { years }\end{array}$ & Normal $^{B}$ & $\begin{array}{l}\text { Before } \\
\text { puberty }\end{array}$ & & $-10^{\circ} \mathrm{C}$ & $\begin{array}{l}0,007 / \\
0.03\end{array}$ & 2 & & \\
\hline RP1539* & p.R1295X/p.N3894D\# & 1987 & USH2 & 20 & $\begin{array}{l}\text { Congenital, } \\
\text { moderate and } \\
\text { stable }\end{array}$ & Normal $^{B}$ & 20 & 17 & $\begin{array}{c}\text { Concentric loss } \\
\text { (20 years) }\end{array}$ & & 2 & & \\
\hline RP1172* & $\begin{array}{c}\text { c.10272_10273dupTT/p. } \\
\text { W2618X }\end{array}$ & 1964 & USH2 & 23 & $\begin{array}{c}\text { Moderate and } \\
\text { stable since } 7 \text { years }\end{array}$ & Normal $^{B}$ & 23 & 18 & $\begin{array}{l}\text { Concentric loss } \\
\text { (38 years) }\end{array}$ & & & $\begin{array}{c}\text { No } \\
\text { response } \\
\text { (39 years) }\end{array}$ & \\
\hline RP1641* & p.C759F/p.W4725X & 1967 & USHA & & $\begin{array}{l}\text { Moderate and } \\
\text { progressive }\end{array}$ & $\begin{array}{c}\text { Central } \\
\text { vestibular } \\
\text { pathology }{ }^{\mathrm{A}}\end{array}$ & 18 & 22 & $\begin{array}{l}\text { Marked } \\
\text { concentric } \\
\text { loss }\end{array}$ & $0,1 / 0,2$ & 2 & $\begin{array}{c}\text { No } \\
\text { response }\end{array}$ & $\mathrm{BE}$ \\
\hline RP1667* & $\begin{array}{c}\text { p.C759F/c.11548 + 2T } \\
>\mathrm{G}\end{array}$ & 1954 & USH2 & 15 & $\begin{array}{l}\text { Mild and slightly } \\
\text { progressive }\end{array}$ & Normal $^{B}$ & 15 & 8 & & & & & $\begin{array}{l}\mathrm{RE}(30 \\
\text { years) }\end{array}$ \\
\hline RP690M* & $\begin{array}{c}\text { p.P4818L/p.Q3368X + } \\
\text { c.5278delG }\end{array}$ & 1972 & USH2 & 22 & $\begin{array}{l}\text { Congenital, } \\
\text { moderate and } \\
\text { stable }\end{array}$ & Normal $^{A}$ & 22 & 8 & & $\begin{array}{c}0,3 / 0,3 \\
\text { (32 years) }\end{array}$ & 1 & $\begin{array}{c}\text { No } \\
\text { response } \\
\text { (31 years) }\end{array}$ & $\begin{array}{l}\mathrm{BE}(25 \\
\text { years })\end{array}$ \\
\hline RP532 & p.Y506X/p.Q3587X & 1968 & USH2 & & Profound & Normal $^{A}$ & 15 & 20 & $\begin{array}{c}\text { Marked } \\
\text { concentric loss }\end{array}$ & $0,1 / 0,1$ & 2 & $\begin{array}{c}\text { No } \\
\text { response }\end{array}$ & $\mathrm{BE}$ \\
\hline RP946* & p.Q3587X/p.E2496E & 1982 & USHNC & 17 & & & & & & & & & \\
\hline RP1613 & c.9260delT/p.G44R & 1971 & USH2 & 30 & Severe & Normal $^{A}$ & 20 & 20 & $\begin{array}{c}\text { Marked } \\
\text { concentric loss }\end{array}$ & $0,1 / 0,6$ & 2 & $\begin{array}{l}\text { Abnormal } \\
\text { response }\end{array}$ & $\mathrm{BE}$ \\
\hline RP1615 & $\begin{array}{c}\text { c.11566delA/c.15053- } \\
\text { 1G > A }\end{array}$ & 1975 & USH2 & 27 & $\begin{array}{l}\text { Moderate since } \\
\text { infancy }\end{array}$ & Normal $^{A}$ & 24 & 25 & $\begin{array}{c}\text { Marked } \\
\text { concentric loss }\end{array}$ & $0,5 / 0,5$ & 2 & $\begin{array}{l}\text { No } \\
\text { response }\end{array}$ & $\mathrm{BE}$ \\
\hline
\end{tabular}

*Parental origin of the mutations was determined; "Patients with possibly pathogenic mutations (UV3).

Age of diagnosis, onset of night blindness and visual field loss are expressed in years.

${ }^{A}$ : results of clinical examinations; ${ }^{B}$ : self reported symptoms.

Eye fundus 1: Bone spicules deposits, attenuation of vessels and waxy pollar of the optic nerve head. Eye fundus 2: $1+$ macular affectation.

ERG: Electroretinography; BE: Both Eyes; LE: Left Eye; RE: Right Eye.

Patients clinically classified as USHA or USHNC are highlighted in bold. 
Table 5 Novel intronic variants

\begin{tabular}{|c|c|c|c|}
\hline Nucleotide change & Intron & $\begin{array}{l}\text { Predicted } \\
\text { pathology }\end{array}$ & $\begin{array}{l}\text { No. of } \\
\text { alleles }\end{array}$ \\
\hline c. $1328+52 T>C$ & IVS7 & - & 3 \\
\hline c. $1841-61 G>A$ & IVS10 & - & 11 \\
\hline c. $4627+32 G>T$ & IVS21 & UV2 & 1 \\
\hline c. $6485+18 C>T$ & IVS33 & UV2 & 1 \\
\hline c.6486-54T > C & IVS33 & UV2 & 2 \\
\hline c.6486-43T > A & IVS33 & UV2 & 1 \\
\hline c. $6657+29 C>A$ & IVS34 & UV2 & 1 \\
\hline c. $8681+18 \mathrm{~A}>\mathrm{G}$ & IVS43 & UV2 & 1 \\
\hline$c .8681+53 T>G$ & IVS43 & UV2 & 1 \\
\hline $\mathrm{C} .8681+118 \mathrm{~A}>\mathrm{G}$ & IVS43 & UV2 & 1 \\
\hline c. $9056-52 \mathrm{G}>\mathrm{T}$ & IVS45 & UV & 1 \\
\hline c. $9372-50 A>G$ & IVS47 & UV2 & 1 \\
\hline c. $9740-59 G>A$ & IVS49 & UV2 & 1 \\
\hline c. $.9958+128 \mathrm{~A}>\mathrm{G}$ & IVS50 & - & 4 \\
\hline c.10388-123T > C & IVS52 & UV2 & 1 \\
\hline c.13812-78A > G & IVS63 & UV2 & 1 \\
\hline c. $14134-53 T>C$ & IVS64 & UV2 & 1 \\
\hline c. $14343+36 G>C$ & IVS65 & UV2 & 1 \\
\hline c.15298-35T > A & IVS70 & UV2 & 1 \\
\hline $\begin{array}{c}\text { c.15298-1153G > A(g.798209G } \\
>\text { A) d }\end{array}$ & IVS71 & UV2 & 1 \\
\hline
\end{tabular}

UV2: probably non- pathogenic mutations; - : neutral variants.

No. of alleles: Number of alleles identified in patients.

${ }^{\mathrm{d}}$ The intronic variant $\mathrm{g} .798209 \mathrm{G}>\mathrm{A}$ affects the last nucleotide of the cochlea specific exon 71. g.DNA numbering starts at nucleotide position 1 in Human Refseq: NG_009497.1 Gl:222352133, which represents the minus (-) strand of USH2A.

in the $U S H 2 A$ gene sequence. According to computational analysis, most of these novel variants were classified as possibly non-pathologic (UV2). (See Table 4).

\section{Discussion}

In the present study, we have performed a wide mutational screening of the $U S H 2 A$ gene in 88 unrelated
Spanish patients diagnosed with Usher syndrome. This analysis has led us to identify a total of 37 different pathogenic mutations, 23 of which had not been previously described: six nonsense, eleven deletions/insertions, two missense, three splice-site mutations and one isocoding variant. At least one mutation was identified in 43 cases and the two responsible mutations were detected in 25 patients (five homozygous and 20 compound heterozygous cases).

The genotype-phenotype correlation for those patients bearing two mutations is illustrated in Table 5. Most cases presented with classical USH2 clinical features. But, interestingly, in one patient (RP-259), the sensorineural hearing loss was profound, RP started at the age of 6 years and he also had vestibular dysfunction (clinical findings typical for USH1). In another intriguing case, phenotype manifestations started at the age of 50 years (RP-1703). We cannot discard the possibility that additional changes in USH $2 \mathrm{~A}$ or in other USH genes, present in these patients, have some modifying effect on the phenotype $[37,38]$.

It is complicated to predict the consequences of missense, silent and intronic changes, in order to discriminate neutral variants from those with a pathogenic effect. We have used a number of bioinformatics' tools to predict the damaging effect of these variants. However, we must bear in mind that these results are only computing predictions and additional studies are necessary to confirm the effect of those changes not clearly classified. In this sense, in vitro analyses for two variants located at non canonical splice sites which were predicted to affect the splicing (p.E2496E and p.V382M) showed that p.E2496E creates a de novo donor splice site stronger than the wild type site that leads to the loss of the last 106 nucleotides of exon 40. Thus, we have considered it as pathogenic. On the other hand, the presence of p.V382M revealed a mild increase of the transcript excluding exon 7 (Figure 1, band d) when the variant was present, but still, the normal transcript has a

Table 6 Results from the three different analysis programs used to predict the pathogenicity of novel missense changes

\begin{tabular}{cccc}
\hline & SIFT & PolyPhen & PMUT \\
\hline p.G44R & Affect (Score 0.01) & Probably damaging & Pathogenic (NN output 0.5113) \\
\hline p.N2377S & Tolerated (Score 0.44) & Possibly damaging & Neutral (NN output 0.2372) \\
\hline p.N2394K & Tolerated (Score 0.23) & Possibly damaging & Neutral (NN output 0.5113) \\
\hline p.G3546R & Affect (Score 0.01) & Probably damaging & Pathogenic (NN output 0.1799) \\
\hline p.N3894D & Tolerated (Score 0.05) & Probably damaging & Neutral (NN output 0.0522) \\
\hline p.E4921K & Tolerated (Score 0.85) & Benign & Neutral (NN output 0.3130) \\
\hline
\end{tabular}

SIFT: SIFT Score ranges from 0 to 1 . The amino acid substitution is predicted to be damaging if the score is $<0.05$, and tolerated if the score is $0 />0.05$. PolyPhen: "Probably damaging" (it is believed most likely to affect protein function or structure), "Possibly damaging" (it is believed to affect protein function or structure), "Benign" (most likely lacking any phenotypic effect).

PMUT: NN output is the original output from the neural network for this mutation and its parameters. If that output is bigger than 0.5 it is predicted as pathogenic, otherwise as neutral. 


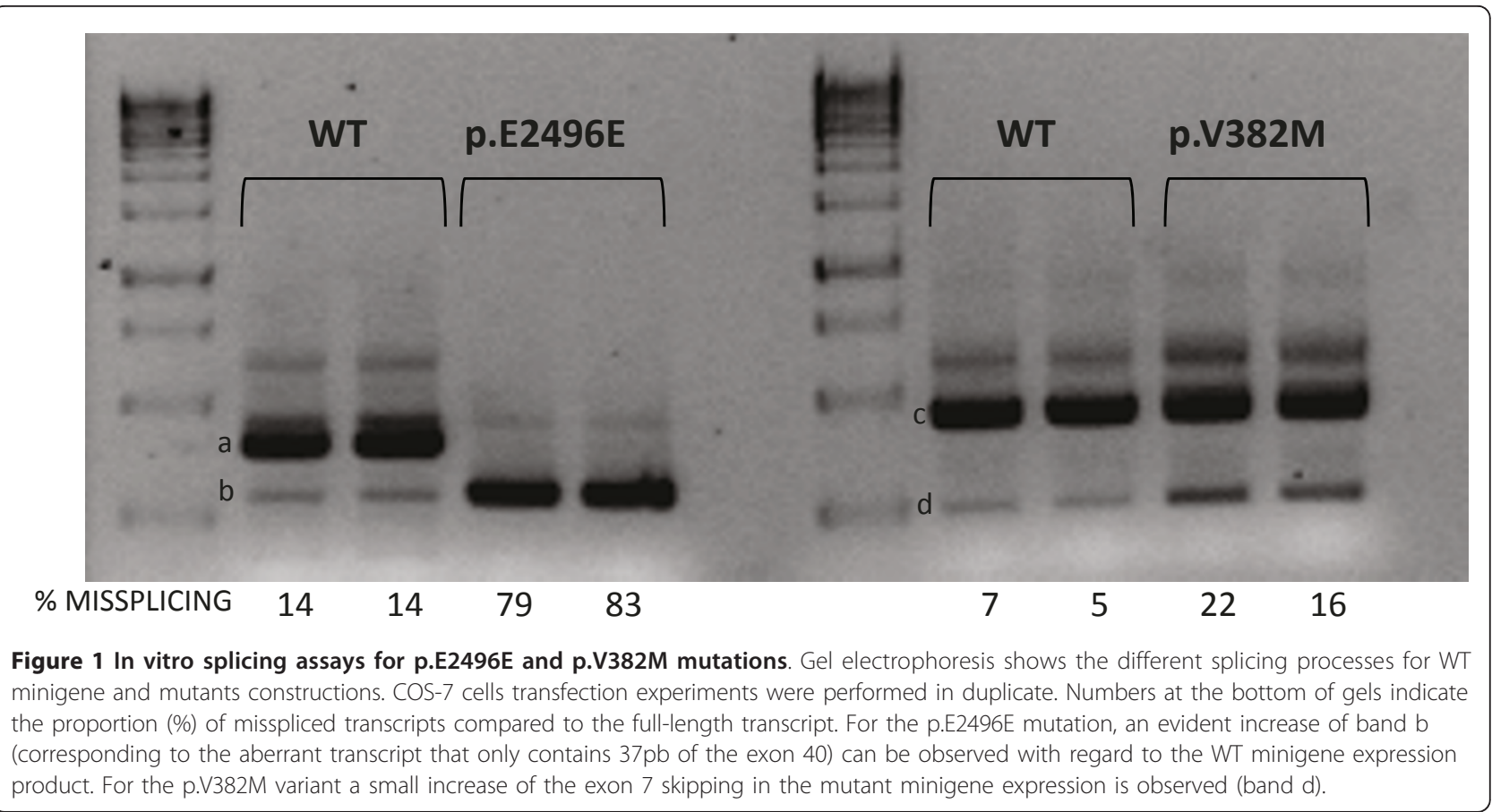

stronger expression. For this reason, this change has been classified as UV3.

The majority of mutations were found once or twice. Only the c.2299delG mutation was identified in more than 5 alleles. However, the cohort in our study is biased, because those patients in whom two mutations were detected in previous analyses (study of exon 13, exons 221 or microarray analyses) were not included in this work. Actually, the allele frequency of the c.2299delG mutation in the Spanish population is $15 \%$, which is lower, in any case, than in other populations [39].

Figure 2 shows the distribution of all the pathogenic mutations detected in the present study, along the different domains of the USH2A protein. Mutations are located evenly throughout the protein and no "hot spots" were observed. Interestingly, there are two domains in which mutations are not detected: the transmembrane and intracellular domains. None of the studies performed in $U S H 2 A$ have detected mutations in the intracytoplasmatic region, involved in the interaction of the USH2A protein with harmonin and whirlin.

There are more than 160 pathogenic variants described in previous studies. Noteworthy, 23 mutations reported in this work are novel. If we compare the mutations detected in this study with those found in other Caucasian populations, only five mutations are common with the studies of Baux et al. (2007) [25] and McGee et al. (2010) [30] and three with the work of Dreyer et al. (2008) [28]. However, we can also find similarities with other populations, like non-Ashkenazi Jews. The splice- site variant c.12062-2A > G was detected in three patients, in homozygous state in one of them. This mutation was initially described by Auslender et al. (2008) [26], as one of the most $U S H 2 A$ prevalent mutations in non-Ashkenazi Jews. Later, it was also detected in the American population [30]. We do not know the origin of our three patients, but it is tempting to speculate that they are descendant of those Sephardic Jews that were expelled from Spain in 1492 [40].

We did not find any mutation in 45 families while in 18 the second mutation remained unidentified. The number of detected pathogenic variants is probably underestimated, because there may be mutations in regions which have not been analyzed (introns, 3' and 5' untranslated regions (UTRs), promoter region, distant enhancers...) or large insertions, deletions and rearrangements that cannot be detected with the conventional PCR techniques. Moreover, some of these patients may have mutations in other genes like GPR98, which seems to be responsible for approximately $3-6 \%$ of USH2 cases $[41,42]$ or $D F N B 31$, although the studies indicate a minor role of DFNB31 in USH2 [43,44]. Furthermore, USH1 genes may be responsible for phenotypically USH2 patients. Jaijo et al. (2010) [32] found two mutations in $\mathrm{CDH} 23$ in two patients diagnosed as USH2 and a high phenotypic heterogeneity due to $C D H 23$ variants has been reported $[45,46]$.

In this report, we have detected at least one mutation in $48.9 \%$ (43/88) of total patients. Considering only the patients clearly diagnosed with Usher syndrome type II, 


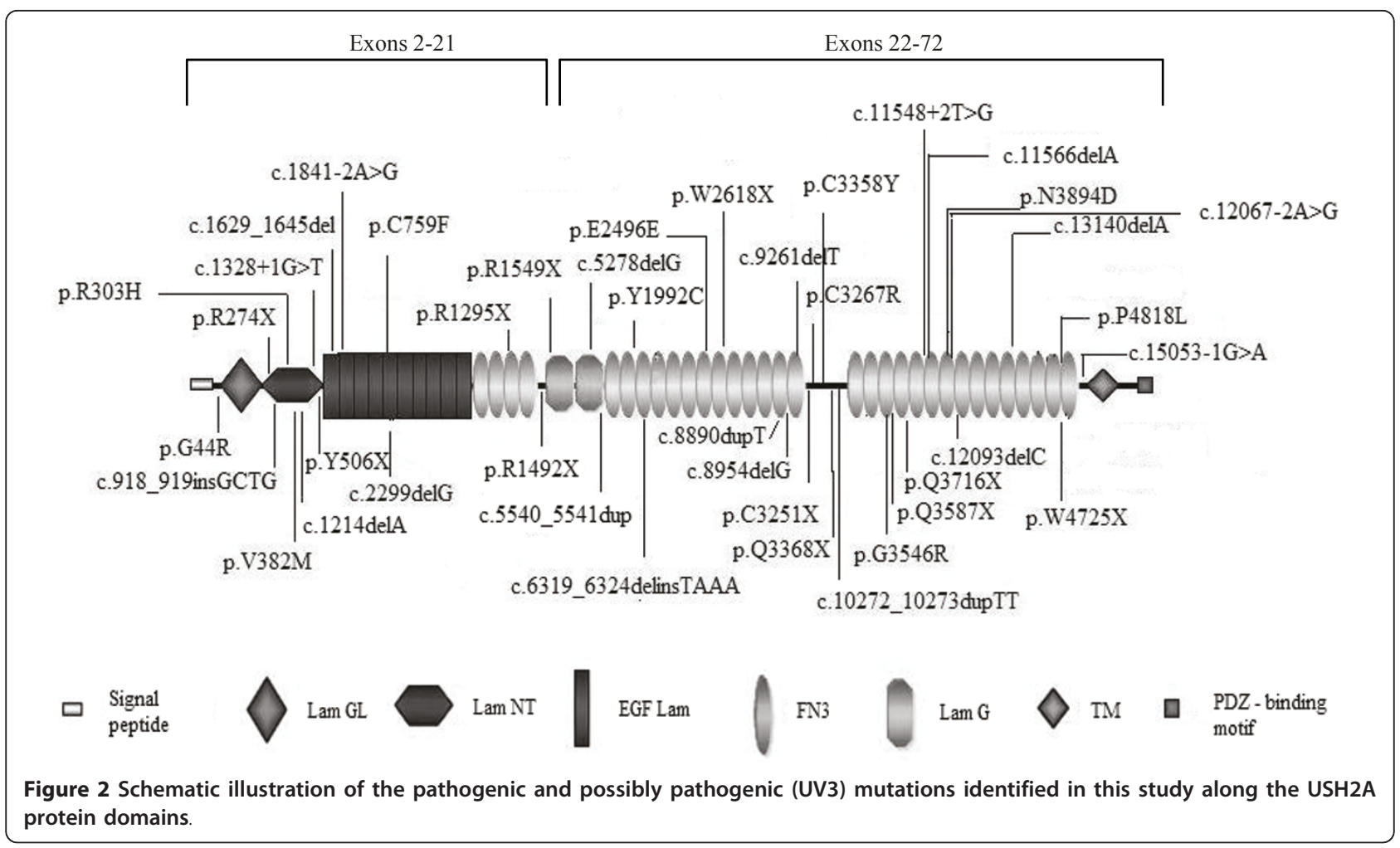

Table 7 Comparison between results obtained from the sample included in the present report with the global results for our total series.

\begin{tabular}{|c|c|c|c|c|c|c|c|c|}
\hline & \multicolumn{4}{|c|}{ PRESENT REPORT SAMPLE } & \multicolumn{4}{|c|}{ TOTAL SERIES ${ }^{\#}$} \\
\hline & 0 MUT & 1 MUT & 2 MUT & $1+2$ MUT $^{*}$ & 0 MUT & 1 MUT & 2 MUT & $1+2$ MUT $^{*}$ \\
\hline USH2 & $43.1 \%(25 / 58)$ & $22.4 \%(13 / 58)$ & $34.5 \%(20 / 58)$ & $56.9 \%(33 / 58)$ & $23.9 \%(32 / 134)$ & $16.4 \%(22 / 134)$ & $59.7 \%(80 / 134)$ & $76.1 \%(102 / 134)$ \\
\hline USHA & $54.5 \%(6 / 11)$ & $18.2 \%(2 / 11)$ & $27.3 \%(3 / 11)$ & $45.5 \%(5 / 11)$ & $33.3 \%(8 / 24)$ & $25 \%(6 / 24)$ & $41.6 \%(10 / 24)$ & $66.7 \%(16 / 24)$ \\
\hline USHNC & $73.7 \%(14 / 19)$ & $15.8 \%(3 / 19)$ & $10.5 \%(2 / 19)$ & $26.3 \%(5 / 19)$ & $64 \%(16 / 25)$ & $12 \%(3 / 25)$ & $24 \%(6 / 25)$ & $36 \%(9 / 25)$ \\
\hline TOTAL & $51.1 \%(45 / 88)$ & $20.5 \%(18 / 88)$ & $28.4 \%(25 / 88)$ & $48.9 \%(43 / 88)$ & $30.6 \%(56 / 183)$ & $16.9 \%(31 / 183)$ & $52.5 \%(96 / 183)$ & $69.4 \%(127 / 183)$ \\
\hline
\end{tabular}

We consider as a mutation (MUT) only those clearly pathogenic. If we also consider UV3, the total figures in the present report sample are $51.1 \%$ (45/88); $17 \%$ (15/88); 31.9\% (28/88) and 48.9\% (43/88) for 0 MUT, 1 MUT, 2 MUT and $1+2$ MUT respectively.

* Percentage of patients with at least one mutation in USH2A: patients with only one mutation + patients with 2 mutations (1MUT + 2MUT).

\# TOTAL SERIES represents the global results obtained from this, together with previous studies [19,9,23,32, unpublished data and present work]

the mutation detection ratio raises to $56.9 \%(33 / 58)$. This detection rate is lower than expected because, as it has been mentioned before, the patient sample included in this study is biased. Thus, if we take into account all our USH2 patients studied so far (including results from previous studies [[19,9,23,32], unpublished data] and the present work), our database includes 102 typical USH2 patients with at least one mutation detected in the USH $2 \mathrm{~A}$ gene and 32 typical USH2 patients who have been studied for all exons of this gene and no mutation was found (Table 7). Thereby, our mutation detection rate rises considerably to $76.1 \%(102 / 134)$, making our percentage similar to those obtained by Baux et al. (2007) [25] and Dreyer et al. (2008) [28].

\section{Acknowledgements}

Authors are grateful to the patients participating in the study and to their family members, and also to the FARPE for their help and co-operation. The exon trapping expression vector PSPL3 was kindly provided by S. TufferyGiraud and I. Bottillo. Funding: This work was supported by PI07/0558, PI08/ 90311 and PI10/01825, from the Spanish Ministry of Science and Innovation and GVPRE/2008/024 from the Conselleria de Sanitat de la Comunitat Valenciana. CIBERER is an initiative of the Institute of Health Carlos III from the Spanish Ministry of Science and Innovation. Gema García-García and María José Aparisi are recipient of a fellowship from the Spanish Ministry of Education (REF: AP2008-02760 and AP2009-3344, respectively). Regina Rodrigo has a Contrato-Investigador SNS Miguel Servet (CP09/118) from Instituto de Salud Carlos III, Ministerio de Ciencia e Innovación. Patient consent: obtained; Ethics approval: This study was conducted according to 
the tenets of Helsinki declaration and with the approval of the Hospital La Fe ethics committee.

\section{Author details}

${ }^{1}$ Grupo de Investigación en Enfermedades Neurosensoriales. Instituto de Investigación Sanitaria IIS-La Fe, Valencia, Spain. ${ }^{2}$ CIBER de Enfermedades Raras (CIBERER), Valencia, Spain. ${ }^{3}$ Servicio de Genética, Fundación Jiménez Díaz, Madrid, Spain. ${ }^{4}$ Servei de Genètica, Hospital de la Santa Creu i Sant Pau, Barcelona, Spain. ${ }^{5}$ Instituto de Microcirugía Ocular, Barcelona, Spain. ${ }^{6}$ Servicio de Oftalmología, Hospital Universitario La Fe, Valencia, Spain ${ }^{7}$ Unidad de Genética y Diagnóstico Prenatal, Hospital Universitario La Fe, Valencia, Spain.

\section{Authors' contributions}

GG carried out the mutational screening of the USH2A gene in Usher syndrome patients, participated in the minigene constructions and expression and drafted the manuscript. MA carried out the mutational screening of the USH2A gene in controls and participated in the minigene constructions and expression. TJ carried out the Splice-site predictions and drafted the manuscript. RR carried out bioinformatics' predictions of the pathogenic effect of missense variations. AL participated in the mutational screening of the USH2A gene in Usher syndrome patients. AA and SB participated in patients' and controls' genomic DNA extraction. FB participated in clinical anamnestic data collection. RN, MB and CA participated in patient recruitment, pedigree and clinical anamnestic data collection. MD participated in the clinical diagnosis of patients. JM participated in patient recruitment, pedigree and clinical anamnestic data collection and drafted the manuscript. EA coordinated and supervised the study and drafted the manuscript. All authors read and approved the manuscript.

\section{Competing interests}

The authors declare that they have no competing interests.

Received: 4 April 2011 Accepted: 17 October 2011

Published: 17 October 2011

\section{References}

1. Keats BJ, Corey DP: The usher syndromes. Am J Med Genet 1999, 89:158-66, Review.

2. Espinós C, Millán JM, Beneyto M, Nájera C: Epidemiology of Usher syndrome in Valencia and Spain. Community Genet 1998, 1:223-8.

3. Saihan Z, Webster AR, Luxon L, Bitner-Glindzicz M: Update on Usher syndrome. Curr Opin Neurol 2009, 22:19-27.

4. Millán JM, Aller E, Jaijo T, Blanco-Kelly F, Gimenez-Pardo A, Ayuso C: An update on the genetics of usher syndrome. J Ophthalmol 2011, 2011:417217.

5. Rosenberg T, Haim M, Hauch AM, Parving A: The prevalence of Usher syndrome and other retinal dystrophy-hearing impairment associations. Clin Genet 1997, 51:314-21.

6. Spandau UH, Rohrschneider K: Prevalence and geographical distribution of Usher syndrome in Germany. Graefes Arch Clin Exp Ophthalmol 2002, 240:495-498.

7. Pieke-Dahl S, van Aarem A, Dobin A, Cremers CW, Kimberling WJ: Genetic heterogeneity of Usher syndrome type II in a Dutch population. J Med Genet 1996, 33:753-7.

8. Rivolta C, Sweklo EA, Berson EL, Dryja TP: Missense mutation in the USH2A gene: association with recessive retinitis pigmentosa without hearing loss. Am J Hum Genet 2000, 66:1975-1978.

9. Aller E, Najera C, Millan JM, Oltra JS, Perez-Garrigues H, Vilela C, Navea A, Beneyto M: Genetic analysis of 2299delG and C759F mutations (USH2A) in patients with visual and/or auditory impairments. Eur J Hum Genet 2004, 12:407-10.

10. Kimberling WJ, Weston MD, Möller C, van Aarem, Cremers CWRJ, Sumegi J, Ing PS, Connolly C, Martini A, Milani M, Tamayo ML, Bernal J, Greenberg J, Ayuso $C$ : Gene mapping of Usher syndrome type lla: localization of the gene to a $2.1-\mathrm{cM}$ segment on chromosome 1q41. Am J Human Genet 1995, 56:216-223.

11. Eudy JD, Weston MD, Yao S, Hoover DM, Rehm HL, Ma-Edmonds M, Yan D, Ahmad I, Cheng JJ, Ayuso C, Cremers C, Davenport S, Moller C, Talmadge CB, Beisel KW, Tamayo M, Morton CC, Swaroop A, Kimberling WJ,
Sumegi J: Mutation of a gene encoding a protein with extracellular matrix motifs in Usher syndrome type lla. Science 1998, 280:1753-7.

12. Weston MD, Eudy JD, Fujita S, Yao S, Usami S, Cremers C, Greenberg J, Ramesar R, Martini A, Moller C, Smith RJ, Sumegi J, Kimberling WJ: Genomic structure and identification of novel mutations in usherin, the gene responsible for Usher syndrome type Ila. Am J Hum Genet 2000, 66:1199-210.

13. van Wijk E, Pennings RJ, te Brinke $H$, Claassen A, Yntema HG, Hoefsloot LH, Cremers FP, Cremers CW, Kremer $\mathrm{H}$ : Identification of 51 novel exons of the Usher syndrome type $2 \mathrm{~A}(\mathrm{USH} 2 \mathrm{~A})$ gene that encode multiple conserved functional domains and that are mutated in patients with Usher syndrome type II. Am J Hum Genet 2004, 74:738-44.

14. Adato A, Lefevre G, Delprat B, Michel V, Michalski N, Chardenoux S, Weil D, El-Amraoui $A$, Petit C: Usherin, the defective protein in Usher syndrome type IIA, is likely to be a component of interstereocilia ankle links in the inner ear sensory cells. Hum Mol Genet 2005, 14:3921-32.

15. van Wijk E, van der Zwaag B, Peters T, Zimmermann $U$, Te Brinke $H$, Kersten FF, Märker T, Aller E, Hoefsloot LH, Cremers CW, Cremers FP, Wolfrum U, Knipper M, Roepman R, Kremer H: The DFNB31 gene product whirlin connects to the Usher protein network in the cochlea and retina by direct association with USH2A and VLGR1. Hum Mol Genet 2006, 15(5):751-65.

16. Liu XZ, Hope C, Liang CY, Zou JM, Xu LR, Cole T, Mueller RF, Bundey S, Nance W, Brown SD: A mutation (2314delG) in the Usher syndrome type IIA gene: high prevalence and phenotypic variation. Am J Hum Genet 1999, 64:1221-1225.

17. Adato A, Weston MD, Berry A, Kimberling WJ, Bonne-Tamir : A Three novel mutations and twelve polymorphisms identified in the USH2A gene in Israeli USH2 families. Hum Mutat 2000, 15:388.

18. Dreyer $B$, Tranebjaerg $L$, Rosenberg $T$, Weston MD, Kimberling WJ, Nilssen Identification of novel USH2A mutations: implications for the structure of USH2A protein. Eur J Hum Genet 2000, 8:500-506.

19. Najera C, Beneyto M, Blanca J, Aller E, Fontcuberta A, Millan JM, Ayuso C: Mutations in myosin VIIA (MYO7A) and usherin (USH2A) in Spanish patients with Usher syndrome types I and II, respectively. Hum Mutat 2002, 20:76-77.

20. Bernal S, Ayuso C, Antiñolo G, Gimenez A, Borrego S, Trujillo MJ, Marcos I, Calaf M, Del Rio E, Baiget M: Mutations in USH2A in Spanish patients with autosomal recessive retinitis pigmentosa: high prevalence and phenotypic variation. J Med Genet 2003, 40:e8.

21. Pennings RJ, Te Brinke $H$, Weston MD, Claassen A, Orten DJ, Weekamp H, Van Aarem A, Huygen PL, Deutman AF, Hoefsloot LH, Cremers FP, Cremers CW, Kimberling WJ, Kremer H: USH2A mutational analysis in 70 Dutch families with Usher syndrome type II. Hum Mutat 2004, 24:185.

22. Maubaret C, Griffoin JM, Arnaud B, Hamel CP: Novel mutations in MYO7A and USH2A in Usher syndrome. Ophthalm Genet 2005, 26:25-29.

23. Aller E, Jaijo T, Beneyto M, Nájera C, Oltra S, Ayuso C, Baiget M, Carballo M, Antiñolo G, Valverde D, Moreno F, Vilela C, Collado D, Pérez-Garrigues H, Návea A, Millán JM: Identification of 14 novel mutations in the long isoform of USH2A in Spanish patients with Usher syndrome type II. $J$ Med Genet 2006, 43:e55.

24. Cremers FP, Kimberling WJ, Külm $M$, de Brouwer AP, van Wijk E, te Brinke $H$, Cremers CW, Hoefsloot LH, Banfi S, Simonelli F, Fleischhauer JC, Berger W, Kelley PM, Haralambous E, Bitner-Glindzicz M, Webster AR, Saihan Z, De Baere E, Leroy BP, Silvestri G, McKay GJ, Koenekoop RK, Millan JM, Rosenberg T, Joensuu T, Sankila EM, Weil D, Weston MD, Wissinger B, Kremer $\mathrm{H}$ : Development of a genotyping microarray for Usher syndrome. J Med Genet 2007, 44:153-160.

25. Baux D, Larrieu L, Blanchet C, Hamel C, Ben Salah S, Vielle A, GilbertDussardier B, Holder M, Calvas P, Philip N, Edery P, Bonneau D, Claustress M, Malcolm S, Roux AF: Molecular and In Silico Analyses of the Full-lenth Isoform of Usherin Identify New Pathogenic Alleles in Usher Type II Patients. Hum Mut 2007, 28:781-9.

26. Auslender N, Bandah D, Rizel L, Behar DM, Shohat M, Banin E, AllonShalev S, Sharony R, Sharon D, Ben-Yosef T: Four USH2A founder mutations underlie the majority of Usher syndrome type 2 cases among non-Ashkenazi Jews. Genet Test 2008, 12:289-94.

27. Dai H, Zhang X, Zhao X, Deng T, Dong B, Wang J, Li Y: Identification of five novel mutations in the long isoform of the USH2A gene in Chinese families with Usher syndrome type II. Mol Vis 2008, 4:2067-75. 
28. Dreyer B, Brox V, Tranebjaerg L, Rosenberg T, Sadeghi AM, Möller C, Nilssen O: Spectrum of USH2A mutations in Scandinavian patients with Usher syndrome type II. Hum Mutat 2008, 29(3):451.

29. Yan D, Ouyang X, Patterson DM, Du LL, Jacobson SG, Liu XZ: Mutation analysis in the long isoform of USH2A in American patients with Usher Syndrome type II. J Hum Genet 2009, 54(12):732-8.

30. McGee TL, Seyedahmadi BJ, Sweeney MO, Dryja TP, Berson EL: Novel mutations in the long isoform of the USH2A gene in patients with Usher syndrome type II or non-syndromic retinitis pigmentosa. J Med Genet 2010, 47(7):499-506.

31. Le Guédard-Méreuze $S$, Vaché $C$, Baux $D$, Faugère V, Larrieu L, Abadie $C$, Janecke A, Claustres M, Roux AF, Tuffery-Giraud S: Ex vivo splicing assays of mutations at noncanonical positions of splice sites in Usher genes. Hum Mutat 2010, 31(3):347-55.

32. Jaijo T, Aller E, García-García G, Aparisi MJ, Bernal S, Avila-Fernández A, Barragán I, Baiget M, Ayuso C, Antiñolo G, Díaz-Llopis M, Külm M, Beneyto M, Nájera C, Millán JM: Microarray-based mutation analysis of 183 Spanish families with Usher syndrome. Invest Ophthalmol Vis Sci 2010, 51(3):1311-7.

33. Jaijo T, Aller E, Aparisi MJ, García-García G, Hernan I, Gamundi MJ, Nájera C, Carballo M, Millán JM: Functional analysis of splicing mutations in MYO7A and USH2A genes. Clin Genet 2011, 79(3):282-8.

34. Bottillo I, De Luca A, Schirinzi A, Guida V, Torrente I, Calvieri S, Gervasini C, Larizza L, Pizzuti A, Dallapiccola B: Functional analysis of splicing mutations in exon 7 of NF1 gene. BMC Med Genet 2007, 12(8):4.

35. Baux D, Faugère V, Larrieu L, Le Guédard-Méreuze S, Hamroun D, Béroud C, Malcolm S, Claustres M, Roux AF: UMD-USHbases: a comprehensive set of databases to record and analyse pathogenic mutations and unclassified variants in seven Usher syndrome causing genes. Hum Mutat 2008, 29(8): E76-E87.

36. Aller E: Molecular bases of Usher syndrome. Identifying mutations in known genes. Functional studies searching for candidate genes. PhD thesis Valencia University, Genetic Department; 2007.

37. de Pontual L, Pelet A, Clement-Ziza M, Trochet D, Antonarakis SE, AttieBitach T, Beales PL, Blouin JL, Dastot-Le Moal F, Dollfus H, Goossens M, Katsanis N, Touraine R, Feingold J, Munnich A, Lyonnet S, Amiel J: Epistatic interactions with a common hypomorphic RET allele in syndromic Hirschsprung disease. Hum Mut 2007, 28:790-6.

38. Khanna H, Davis EE, Murga-Zamalloa CA, Estrada-Cuzcano A, Lopez I, den Hollander Al, Zonneveld MN, Othman MI, Waseem N, Chakarova CF, Maubaret C, Diaz-Font A, Macdonald I, Muzny DM, Wheeler DA, Morgan M, Lewis $L R$, Logan CV, Tan PL, Beer MA, Inglehearn CF, Lewis RA, Jacobson SG, Bergmann C, Beales PL, Attié-Bitach T, Johnson CA, Otto EA, Bhattacharya SS, Hildebrandt F, Gibbs RA, Koenekoop RK, Swaroop A, Katsanis N: A common allele in RPGRIP1L is a modifier of retinal degeneration in ciliopathies. Nat Genet 2009, 41(6):739-45.

39. Aller E, Larrieu L, Jaijo T, Baux D, Espinós C, González-Candelas F, Nájera C, Palau F, Claustres M, Roux AF, Millán JM: The USH2A c.2299delG mutation: dating its common origin in a Southern European population. Eur I Hum Genet 2010, 18(7):788-93

40. Millán JM, Aller E, Jaijo T, Blanco-Kelly F, Corton M, Ayuso C: Molecular Epidemiology of Usher syndrome. In Usher syndrome: Pathogenesis, Diagnosis and Therapy. Edited by: Satpal Ahuja. Nova Publishers, Hauppauge NY; 2011:

41. Weston MD, Luijendijk MW, Humphrey KD, Möller C, Kimberling WJ: Mutations in the VLGR1 gene implicate G-protein signaling in the pathogenesis of Usher syndrome type II. Am J Hum Genet 2004, 74:357-66, Erratum in: Am J Hum Genet. 2004:74:1080.

42. Ebermann I, Wiesen MH, Zrenner E, Lopez I, Pigeon R, Kohl S, Löwenheim H, Koenekoop RK, Bolz HJ: GPR98 mutations cause Usher syndrome type 2 in males. J Med Genet 2009, 46(4):277-80.

43. Ebermann I, Scholl HP, Charbel Issa P, Becirovic E, Lamprecht J, Jurklies B, Millán JM, Aller E, Mitter D, Bolz H: A novel gene for Usher syndrome type 2: mutations in the long isoform of whirlin are associated with retinitis pigmentosa and sensorineural hearing loss. Hum Genet 2007, 121:203-11.

44. Aller E, Jaijo T, van Wijk E, Ebermann I, Kersten F, García-García G, Voesenek K, Aparisi MJ, Hoefsloot L, Cremers C, Díaz-Llopis M, Pennings R, Bolz HJ, Kremer H, Millán JM: Sequence variants of the DFNB31 gene among Usher syndrome patients of diverse origin. Mol Vis 2010, 23(16):495-500.
45. Bolz H, von Brederlow B, Ramírez A, Bryda EC, Kutsche K, Nothwang HG, Seeliger M, del C-Salcedó Cabrera M, Vila MC, Molina OP, Gal A, Kubisch C: Mutation of $\mathrm{CDH} 23$, encoding a new member of the cadherin gene family, causes Usher syndrome type 1D. Nat Genet 2001, 27:108-12.

46. Astuto LM, Bork JM, Weston MD, Askew JW, Fields RR, Orten DJ, Ohliger SJ, Riazuddin S, Morell RJ, Khan S, Riazuddin S, Kremer H, van Hauwe P, Moller CG, Cremers CW, Ayuso C, Heckenlively JR, Rohrschneider K, Spandau U, Greenberg J, Ramesar R, Reardon W, Bitoun P, Millan J, Legge R, Friedman TB, Kimberling WJ: CDH23 mutation and phenotype heterogeneity: a profile of 107 diverse families with Usher syndrome and nonsyndromic deafness. Am J Hum Genet 2002, 71(2):262-75.

doi:10.1186/1750-1172-6-65

Cite this article as: Garcia-Garcia et al: Mutational screening of the USH2A gene in Spanish USH patients reveals 23 novel pathogenic mutations. Orphanet Journal of Rare Diseases 2011 6:65.

\section{Submit your next manuscript to BioMed Central and take full advantage of:}

- Convenient online submission

- Thorough peer review

- No space constraints or color figure charges

- Immediate publication on acceptance

- Inclusion in PubMed, CAS, Scopus and Google Scholar

- Research which is freely available for redistribution

Submit your manuscript at www.biomedcentral.com/submit
Ciomed Central 